\title{
Paraprotein-associated diseases: a complex relationship between monoclonal gammopathy of unknown significance and severe organ damage
}

\author{
Hermine Agis
}

Accepted: 18 January 2021

(C) Springer-Verlag GmbH, AT part of Springer Nature 2021

In this special issue we want to introduce new insights into a complex relationship between monoclonal gammopathy of unknown significance (MGUS) [1, 2], monoclonal gammopathy of renal significance (MGRS) [3], immunoglobulin light chain amyloidosis (AL) [4] and monoclonal gammopathy with clinical significance (MGCS) [5]. All of them are categorized as $\mathrm{M}$-protein related diseases.

MGUS is a premalignant condition with an incidence of $3 \%$ for persons who are 50 years of age, with the incidence continuously increasing with age. MGUS and smoldering multiple myeloma (SMM) are defined by a measurable paraprotein (M-protein, M-spike or M-gradient), a detectable clonal plasmacell or B-cell population in the bone marrow, and without any related organ damage.

Hematologists and oncologists are trained to be aware of progression from MGUS/SMM to multiple myeloma (MM). In rare cases, the premalignant condition does not progress into malignant uncontrolled cell growth like plasma-cells in MM but the clonal M-protein can acquire a "toxic" character and causes severe tissue damage or life-threatening organ dysfunction. It is important to realize that the severity of the injury is independent of the M-protein concentration. Very low levels of M-protein can induce severe and highly dangerous organ dysfunction.

In recent years, knowledge about the diverse categories of M-protein induced organ damages has been continuously growing. Intensive efforts have been made to improve awareness and clarify the complex

\footnotetext{
H. Agis, MD (ه)

Division of Hematology and Hemostaseology,

Department of Internal Medicine I, Medical

University Vienna and General Hospital Vienna,

Waehringer Guertel 18-20, 1090 Vienna, Austria

hermine.agis@meduniwien.ac.at
}

mechanisms of tissue and organ damage by a "toxic" M-protein. Delayed diagnosis and prolonged treatment-initiation often result in loss of organ function and may cause life-threatening systemic deterioration.

If there is suspicion of M-protein-induced tissue damage, the relationship between $M$-protein and organ dysfunction has to be clearly defined. The next most important step is timely treatment initiation to avoid irreversible long-term organ damage. In summary, awareness and early treatment are of pivotal importance for improvement of overall survival and restoration of organ function. These rare and complex diseases are summarized within two new concepts named monoclonal gammopathy of renal significance (MGRS) and monoclonal gammopathy of clinical significance (MGCS).

AL amyloidosis is an extensively studied rare disease. The mechanism of tissue damage is almost clarified. The clonal cell population produces a misfolded light-chain, forming insoluble extracellular fibrils and building amyloid depositions.

MGRS represents a very new entity within this concept that is currently of great research interest. The growing information about these complex interactions clearly supports and influences the awareness and daily routine in medical care of MGUS and SMM patients. It is important to note that by implementing simple biomarkers into the clinical routine parameter-checklist, organ function and even lives might be saved.

Due to the fact that final diagnosis and treatment initiation are based on an intensive workup including interdisciplinary collaboration between multiple disciplines (hematologists, nephrologists, cardiologists, pathologists, radiologists), patients with suspected paraprotein-associated tissue damage should be sent to a highly experienced center. 
With the intention to highlight the urgent need in awareness and early diagnosis of these rare diseases and to outline the complexity and intensive collaboration needed for diagnosis and treatment, we present a special edition of paraprotein-associated diseases.

Conflict of interest H. Agis: $A M G E N$ : Honoraria for Advisory Board Participation. Bristol-Myers Squibb (BMS): Honoraria for Advisory Board Participation. Celgene: Honoraria and Research Funding. Janssen: Honoraria and Research Funding. Sanofi: Honoraria for Advisory Board Participation. Takeda: Honoraria and Research Funding.

\section{References}

1. Kyle RA, Durie BG, Rajkumar SV, et al. Monoclonal gammopathy of undetermined significance (MGUS) and smoldering (asymptomatic) multiple myeloma: IMWG consensus perspectives risk factors for progression and guidelines for monitoring and management. Leukemia. 2010;24(6):1121-7. https://doi.org/10.1038/leu.2010.60.

2. Merlini G, Stone MJ. Dangerous small B-cell clones. Blood. 2006;108(8):2520-30. https://doi.org/10.1182/blood2006-03-001164.

3. Leung N, Bridoux F, Batuman V, et al. The evaluation of monoclonal gammopathy of renal significance: a con- sensus report of the International Kidney and Monoclonal Gammopathy Research Group. Nat Rev Nephrol. 2019;15(1):45-59. https://doi.org/10.1038/s41581-0180077-4. Erratumin: Nat Rev Nephrol. 2019;15(2):121.

4. Merlini G, Dispenzieri A, Sanchorawala V, et al. Systemic immunoglobulin light chain amyloidosis. Nat Rev Dis Primers. 2018;4(1):38. https://doi.org/10.1038/s41572018-0034-3.

5. Fermand JP, Bridoux F, Dispenzieri A, et al. Monoclonal gammopathy of clinical significance: a novel concept with therapeutic implications. Blood. 2018;132(14):1478-85. https://doi.org/10.1182/blood-2018-04-839480.

Publisher's Note Springer Nature remains neutral with regard to jurisdictional claims in published maps and institutional affiliations.

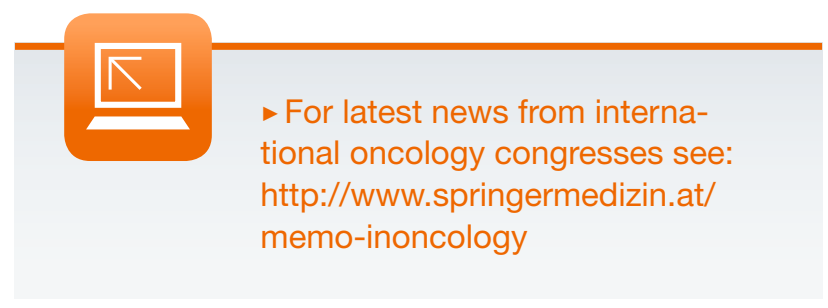

